\title{
Detección de cavitación en una bomba centrífuga usando emisiones acústicas
}

\author{
Cavitation detection in a centrifugal pump using acoustic emissions \\ Jabid Quiroga M. ${ }^{1} \quad$ Silvia Oviedo C. ${ }^{1} \quad$ Alfonso García C. ${ }^{1}$ \\ Recibido 1 de marzo de 2011, aceptado 13 de agosto de 2012 \\ Received: March 1, $2011 \quad$ Accepted: August 13, 2012
}

\begin{abstract}
RESUMEN
En el presente artículo se propone el uso de las emisiones acústicas para el monitoreo de la cavitación en una bomba centrífuga. Este monitoreo se ejecuta a través del seguimiento a unos indicadores de falla obtenidos a partir del valor RMS de la señal de emisiones acústicas en dominio tiempo y el valor RMS de los coeficientes de la Transformada Discreta Wavelet (TDW) usando la onda madre db6 de la misma señal acústica. La experimentación se realiza en un banco dedicado que permite cavitar a una bomba de $1 / 2 \mathrm{hp}$ en distintos niveles de severidad y bajo diferentes condiciones de bombeo. Resultados experimentales mostraron que los indicadores propuestos permiten detectar y evaluar cualitativamente los niveles de severidad de la cavitación en una bomba centrífuga.
\end{abstract}

Palabras clave: Cavitación, monitoreo, detección de fallas, emisiones acústicas, transformada discreta Wavelet.

\begin{abstract}
In this paper an acoustic emission based cavitation fault detection system is proposed for a centrifugal pump. The monitoring is performed tracking a fault indicator obtained using the RMS value of the acoustic emission signal in time domain and the RMS value of the coefficients obtained by applying Discrete Wavelet Transform on the acoustic signal using db6 mother Wavelet. Experiments in different cavitation levels and under different operation conditions are carried out in a $1 / 2 \mathrm{hp}$ centrifugal pump dedicated test bed. Results showed that the proposed fault indicators are suitable for detecting and evaluating cavitation severities in a centrifugal pump.
\end{abstract}

Keywords: Cavitation, monitoring, fault detection, acoustic emissions, discrete Wavelet transform.

\section{INTRODUCCIÓN}

Los sistemas de producción requieren de confiabilidad y disponibilidad en sus equipos y procesos. Las fallas en equipos son inevitables y pueden afectar los sistemas productivos a través de paradas no programadas, disminución de la vida útil de los equipos, altos costos de reparación o reemplazo y afectación del proceso. Por lo tanto, es importante para la industria contar con herramientas que permitan detectar fallas sin necesidad de detener los equipos y, mejor aún, que esta detección se realice en las primeras etapas de la falla.

Dentro de los esquemas de mantenimiento, el Mantenimiento Basado en la Condición (CBM, por sus siglas en inglés) ha tenido un creciente auge, por cuanto en su esquema de operación supervisa continuamente el estado de los equipos, basándose en la información que provee éste en alguna forma, como vibración, sonido, temperatura, entre otras, y emite alarmas cuando alguna condición anormal se presenta aun en sus etapas iniciales.

\footnotetext{
1 Escuela de Ingeniería Mecánica. Universidad Industrial de Santander. Cra. 27 Calle 9. Cuidad Universitaria. Bucaramanga, Colombia.E-mail: jabib@uis.edu.co; siljuovi@uis.edu.co; alfonso@uis.edu.co
} 
Una de las herramientas para el monitoreo de sistemas es el análisis de las emisiones acústicas, el cual recientemente ha incrementado su popularidad [1]. Las emisiones acústicas detectadas en puntos estratégicos del equipo son analizadas para obtener información en el dominio del tiempo o de la frecuencia. En presencia de falla las emisiones acústicas transportan información que puede ser utilizada para detectar condiciones anormales. Estudios han mostrado que las emisiones acústicas han sido efectivas para monitorear turbinas, compresores, generadores y de manera específica para el monitoreo de rodamientos en equipo rotativo [2]. Sin embargo, la detección de la cavitación no es una tarea fácil, porque depende del diseño del equipo que está siendo monitorizado, las condiciones de operación, el tipo de cavitación y localización del fenómeno son diferentes [3].

Por otra parte, las bombas hidráulicas son un equipo esencial en muchos procesos. Actualmente, hay información limitada en el uso de las emisiones acústicas en la detección de la cavitación [4]. En este artículo se presenta un caso de estudio en el cual se han utilizado las emisiones acústicas para detectar la cavitación en una bomba centrífuga de $1 / 2 \mathrm{hp}$ bajo distintos niveles de severidad y bajo distintas condiciones de operación utilizando la información de la señal en dominio tiempo y la TDW. En este esquema de monitoreo se determina de una forma no invasiva la presencia del fenómeno de la cavitación en distintos grados de severidad.

\section{ANÁLISIS TEÓRICO}

\section{Cavitación}

La cavitación es una condición anormal en los sistemas de bombeo que se presenta principalmente en la succión de las bombas. La cavitación se desarrolla en varias etapas. Inicialmente se forman burbujas dentro del líquido cuando este se vaporiza bajo condiciones de baja presión. Posteriormente se produce un crecimiento en el volumen de las burbujas existentes y en el número de nuevas burbujas. Las burbujas son arrastradas desde el ojo del impulsor hacia los álabes y su periferia. Debido a la rotación del impulsor, las burbujas adquieren altas velocidades y se desplazan hacia regiones de alta presión donde empiezan a implosionar. Estudios experimentales y teóricos han mostrado que el colapso de una burbuja producto de la cavitación puede producir un microchorro de alta velocidad, resultando en una eventual erosión en las superficies vecinas, específicamente provocando socavaciones en el impulsor [5].

El fenómeno de cavitación descrito afecta considerablemente el proceso de bombeo y por lo general produce una reducción del caudal bombeado y su presión debido a que el volumen ocupado por el fluido en fase gaseosa reduce el espacio disponible para el líquido a ser bombeado. En casos extremos se puede producir una interrupción total del bombeo. Adicionalmente, la formación y colapso de las burbujas no es uniforme en el interior de la cavidad de la bomba, por lo que se producen fluctuaciones en el flujo. Otra consecuencia de la cavitación es la disminución de la cabeza de descarga en la bomba debido a que se destina una parte de la energía disponible para el bombeo en aumentar la velocidad del líquido empleado para llenar las cavidades que dejan las burbujas colapsadas. [6]. Por lo anterior, el efecto hidráulico de la cavitación en una bomba produce un cambio significativo en su capacidad y en su desempeño.

En cuanto a la sintomatología asociada a la cavitación y que puede monitorearse se tiene la presencia de ciertas frecuencias en el espectro de vibración [7] o la presencia de emisiones acústicas [2]. Estas señales se presentan por las ondas de choque que se producen al momento de la implosión de las burbujas en el seno del fluido $\mathrm{y}$ en la vecindad del impulsor.

La vibración se debe al efecto de cargas disparejas actuando sobre el impulsor y que son generadas por una mezcla no uniforme de líquido y vapor, así como por las ondas de choque por el colapso de las burbujas. La formación y colapso de las burbujas se alternará periódicamente con la frecuencia resultante de la velocidad y número de álabes.

\section{Emisiones acústicas (EA)}

Las emisiones acústicas son ondas elásticas generadas por una rápida transferencia de energía desde una fuente localizada dentro del material y que viajan por éste. Las emisiones acústicas aparecen cuando los materiales se someten a esfuerzos por deformación, agrietamiento y erosión. Estas señales usualmente en el rango de $100 \mathrm{KHz}$ a $1 \mathrm{MHz}$ pueden medirse usando transductores piezoeléctricos [2]. 
Entre las ventajas asociadas al monitoreo usando las EA en máquinas rotodinámicas, se tiene la posibilidad de realizar la detección con el equipo en operación y bajo cualquier condición de carga. Adicionalmente, el ancho de banda de las frecuencias excitadas por la falla por lo general es lejano a las frecuencias propias del proceso y, por último, permite evaluar el grado de severidad de la falla monitoreada y su tendencia. Estudios publicados [8-9] demuestran un enfoque particular en la detección de cavitación usando EA, experimentando con parámetros relacionados a la densidad espectral de potencia de la señal acústica. En este trabajo se presenta un enfoque alterno, usando la transformada discreta de Wavelet.

\section{Transformada Wavelet}

En este artículo se aplica la transformada Wavelet discreta a la señal de emisiones acústicas para la generación de un indicador de falla que permita detectar la presencia de cavitación.

La transformada Wavelet es una técnica de procesamiento de señales que ha sido ampliamente utilizada en monitoreo de sistemas debido a que usa funciones bases finitas y con formas similares a los rasgos de fallas, permite realizar espectros en anchos de banda específicos de manera simultánea y su ortogonalidad permite la ubicación de información en dominio tiempo y frecuencia de forma simultánea.

La transformada Wavelet continua se define en (1) [10-11].

$$
\left(W_{\psi} x\right)_{(b, a)}=\frac{1}{\sqrt{a}} \int_{-\infty}^{\infty} x(t) \psi\left(\frac{t-b}{a}\right) d t=\left\langle x(t), \psi_{a, b}(t)\right\rangle
$$

donde $y(t)$ es la función base "la Wavelet madre". La localización en el tiempo es determinada por el término (2).

$$
\psi_{a, b}(t)=\frac{1}{\sqrt{a}} \psi\left(\frac{t-b}{a}\right)
$$

donde $\psi_{a, b}(t)$ es el conjunto de Wavelets generadas por $\psi(t)$, la cual se expande y atenúa o se comprime y amplifica al incrementar o disminuir $a$ respectivamente. Adicionalmente, $\psi(t)$ produce traslación con los cambio de $b$ en el dominio tiempo.
La Transformada Discreta Wavelet (TDW) se obtiene al considerar los parámetros de escala $a$ y de traslación $b$ como valores discretos: $a=a_{o}^{j}, b=k b_{o} \operatorname{con} j, k \epsilon$ $Z, a_{o}>1$ y $b_{o}>0$. Al reemplazar estos valores en (1) se obtiene (3) [11]:

$$
\left(W_{\psi} x\right)_{(j, k)}=\frac{1}{\sqrt{a_{0}^{j}}} \int_{-\infty}^{\infty} x(t) \psi\left(a_{0}^{-j} t-k b_{0}\right) d t=\left\langle x(t), \psi_{j, k}(t)\right\rangle
$$

Donde el conjunto de funciones Wavelet $\psi_{j, k}(t)$ está dado por (4).

$$
\psi_{j, k}(t)=\frac{1}{\sqrt{a_{0}^{j}}} \psi\left(a_{0}^{-j} t-k b_{0}\right)
$$

Por lo tanto, para una apropiada Wavelet madre y parámetros $a_{o}$ y $b_{o}$ es posible representar la señal $x(t)$ como una combinación de funciones Wavelet $\psi_{j, k}(t)$, escaladas y trasladadas [10].

El algoritmo necesario para implementar la TDW se basa en un banco de filtros FIR pasa-altos y pasa-bajos con una respuesta al impulso $a_{m}$ y $b_{m}$, respectivamente, seguidas de un proceso de decimación por dos. Por lo tanto, si se tienen $c_{n}$ muestras de la señal a la entrada de los filtros, los coeficientes de aproximación $c_{n-1}$ se obtienen a la salida del filtro pasa-bajo y $d_{n-1}$ son los coeficientes de detalle a la salida del filtro pasa-altas (5) [8]. El esquema de descomposición descrito arriba se puede observar en la Figura 1.

$$
c_{n-1, k}=\sum_{m} a_{m-2 k} c_{n, m} ; \quad d_{n-1, k}=\sum_{m} b_{m-2 k} c_{n, m}
$$

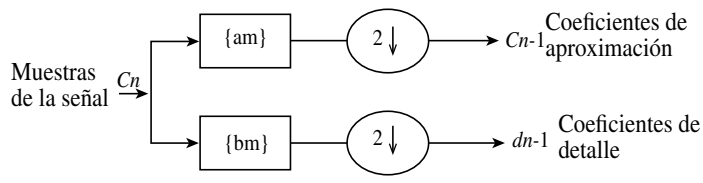

Figura 1. Esquema de descomposición usando Wavelet.

El indicador de falla propuesto en este trabajo basado en la TDW se determina a través del valor RMS a los coeficientes obtenidos cuando la onda madre db6 (Daubechies 6) es aplicada a la señal acústica en dos niveles de descomposición. La selección de la onda madre db6 resultó de una comparación en desempeño 
de una serie de ondas madres ortogonales entre las cuales se tienen la Coiflets, Symlets y la Daubechies. El nivel de descomposición se establece basado en la frecuencia de muestreo de la señal acústica, que en el caso de este estudio fue de $2,5 \mathrm{MHz}$ y en el rango de frecuencia de interés que corresponde a las frecuencias inferiores a $700 \mathrm{KHz}$. Este rango de frecuencia de interés se establece a través de la observación del cambio de la energía espectral cuando la severidad de la cavitación aumenta. Esto indica que la información relativa al fenómeno de cavitación se encuentra en el rango de frecuencias en el que se puedan apreciar cambios cuando la severidad del fenómeno varía.

\section{EXPERIMENTACIÓN}

\section{Banco de pruebas}

Para el estudio del fenómeno de la cavitación y su relación con la señal de emisiones acústicas se implementa un banco dedicado para esta clase de estudios que está compuesto por una bomba centrífuga de $1 / 2 \mathrm{hp}$, un tanque de 30 litros de capacidad adecuado para abastecer la bomba centrífuga y soportar presión de vacío, una bomba de vacío de $1 / 2 \mathrm{hp}$, un sensor piezoeléctrico, un osciloscopio y un computador para el análisis de los datos.

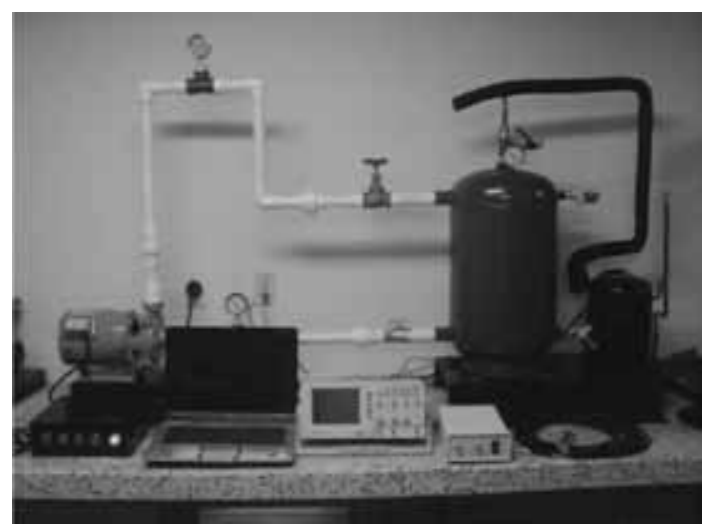

Figura 2. Banco de cavitación.

En la Figura 2 se muestra el banco con todas sus partes. Para la captación de la señal se utiliza un sensor piezoeléctrico que contiene un preamplificador integrado, conectado a una entrada FET de bajo nivel de ruido y un preamplificador de $40 \mathrm{~dB}$.

El procesamiento de la señal se implementa en dos etapas. En la primera se utiliza un osciloscopio que captura la señal de las emisiones acústicas que proviene del sensor piezoeléctrico a una frecuencia de muestreo de 2,5 MHz. Posteriormente, los datos son exportados desde el osciloscopio para procesarlos en ambiente Matlab, como se muestra en el esquema de la Figura 3.

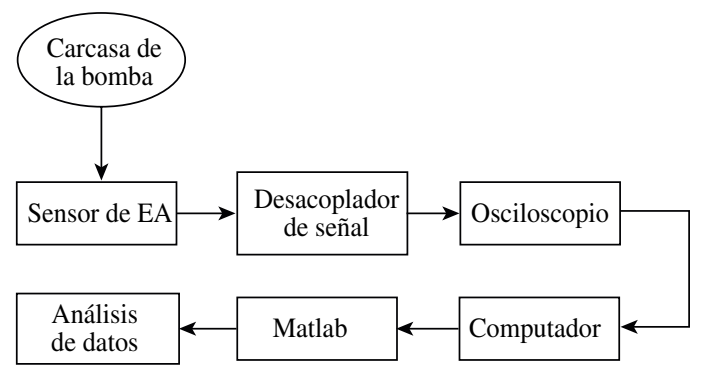

Figura 3. Esquema para captura y procesamiento de señal.

\section{Generación de falla y toma de datos}

Para realizar el estudio propuesto se plantearon tres condiciones de operación de la bomba, las cuales corresponden al $100 \%, 83 \%$ y $60 \%$ del caudal nominal de la bomba. En cada uno de estos escenarios se indujeron dos diferentes niveles de severidad en la cavitación. En cada escenario de prueba se registran la presión de succión $P s$, la presión de descarga $P d$ y la presión en el tanque $P o$; esta última se varía para inducir los diferentes niveles de cavitación. Esta información permite la determinación de la cabeza de bombeo $H$, la cual corresponde a la diferencia entre $P d$ y $P s$, valor usado como referente para determinar la presencia de cavitación incipiente (caída del 3\% de la cabeza de la bomba con respecto a la cabeza en condiciones normales) [6]. Para determinar los demás niveles de severidad de cavitación se calculó una relación entre el comienzo incipiente de la cavitación y la cabeza de la bomba con cavitación (6).

$$
R_{c}=\frac{H_{@(3 \%)}}{H}
$$

En cada escenario se registran los datos correspondientes a la condición normal, cavitación incipiente y cavitación severa. Estos datos se procesan en dominio tiempo usando el RMS de la señal. Adicionalmente se usa la TDW para obtener los coeficientes de la aproximación usando db6 para el respectivo análisis. Cada uno de los anteriores valores se postula como 
posible indicador a ser monitoreado usando las señales acústicas.

El punto de captura de la señal con el sensor piezoeléctrico sobre la superficie de la carcasa de la bomba se determinó en pruebas preliminares. En la Figura 4 se muestran los puntos estudiados en la carcasa y dentro de los cuales el señalado como 1 mostró los mejores resultados en el monitoreo.

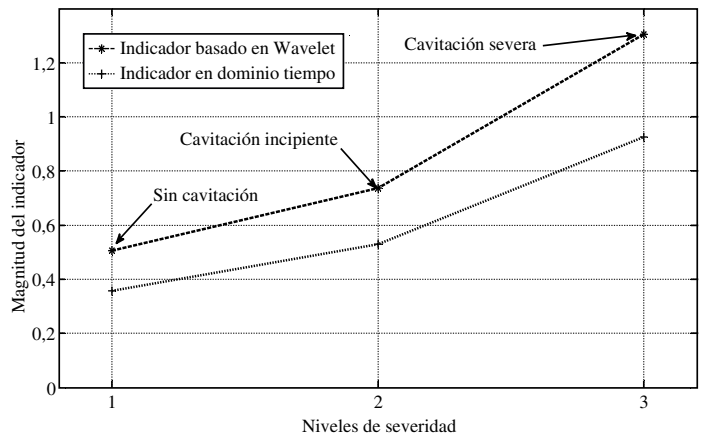

Figura 5. Resultados experimentales para el caso 1.

\section{ANÁLISIS DE DATOS}

\section{Caso $1.100 \%$ del caudal nominal}

En este caso se tienen unas relaciones de severidad $R c$ de 1,02 para severidad leve y 1,35 para severidad crítica calculadas usando (6). En cada escenario de prueba se tomaron siete lecturas. Para cada ensayo se determinan los indicadores de falla propuestos. Las variaciones máximas de los valores pico a pico obtenidas en los diferentes ensayos no superan el $10 \%$. Los valores obtenidos son promediados y se ilustran en la Figura 5.

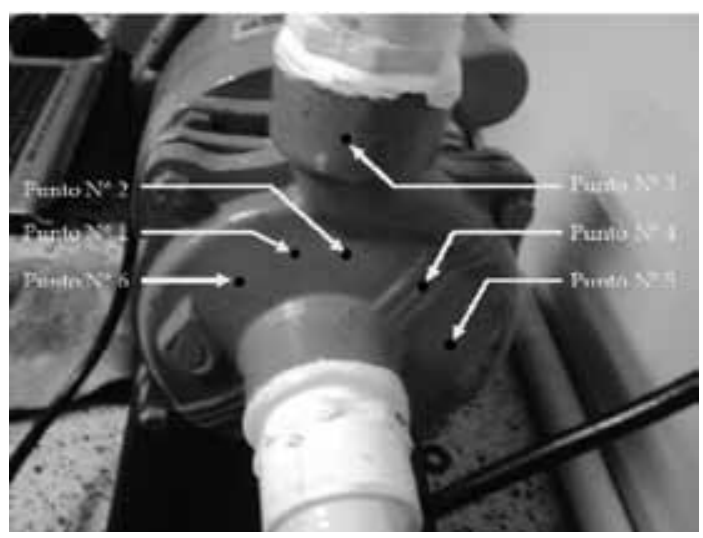

Figura 4. Punto de contacto entre el sensor y la carcasa de la bomba.

\section{Caso $2.83 \%$ del caudal nominal}

Para este caso, el caudal de bombeo es reducido al $83 \%$ del caudal nominal. Al igual que en el caso anterior, se evalúan dos niveles de severidad. Los grados de severidad incipiente y severa en este caso corresponden a las relaciones de 1,13 y 1,56. Para cada escenario de falla se repite la toma de muestras y se promedian los valores en dominio tiempo. El valor promedio es utilizado para generar los indicadores propuestos. En la Figura 6 se puede apreciar el comportamiento de los indicadores propuesto en cada nivel de severidad.

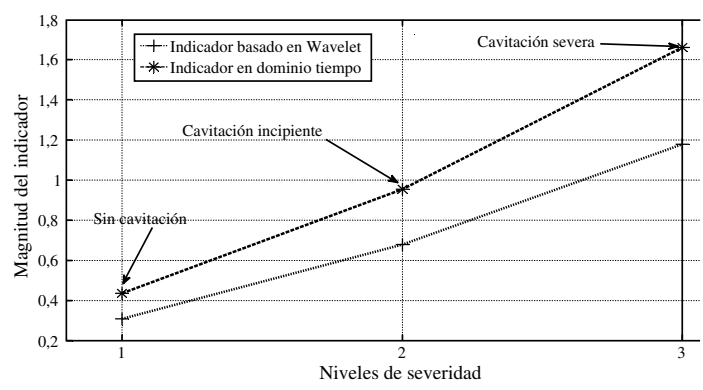

Figura 6. Resultados experimentales para el caso 2 .

\section{Caso 3. $60 \%$ del caudal nominal}

En este último caso, el caudal bombeado se reduce al $60 \%$ del nominal. La experimentación es análoga a los anteriores casos. Las relaciones son de 1,02 y 1,1 para la severidad incipiente y severa respectivamente. Los resultados de los indicadores propuestos se pueden observar en la Figura 7.

En todos los casos estudiados se observa una clara relación entre el RMS en dominio tiempo de la señal de emisiones acústicas y el RMS de los coeficientes de la descomposición usando db6 de la misma señal. En los casos estudiados la magnitud del RMS en dominio tiempo y la magnitud del RMS de los coeficientes de la señal descompuesta usando Wavelet se incrementaron con la severidad de la cavitación. Adicionalmente, se observó que el valor de RMS de ambos indicadores cuando la bomba opera en condición normal en todos los casos se fue incrementando en la medida en que el caudal se redujo. Por lo tanto, el establecimiento de un sistema para monitorear un equipo específico demanda el establecimiento de un umbral determinado a través de la experimentación. 


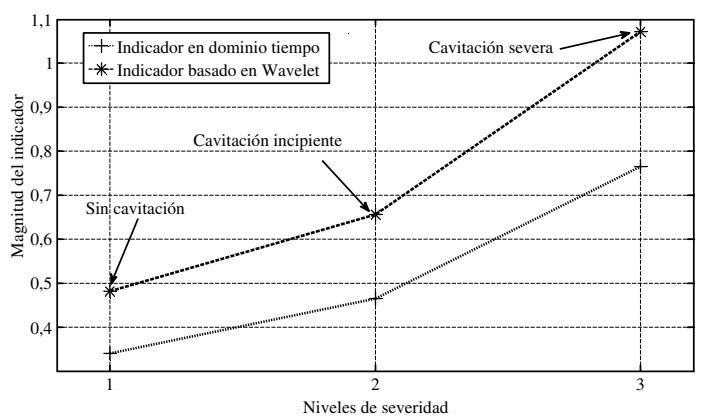

Figura 7. Resultados experimentales para el caso 2 .

La Tabla 1 resume los resultados obtenidos en la experimentación en los diferentes escenarios estudiados.

Tabla 1. Valor de los indicadores propuestos en cada caso estudiado.

\begin{tabular}{|l|c|c|c|c|c|c|}
\hline & \multicolumn{2}{|c|}{ Normal } & \multicolumn{2}{c|}{$\begin{array}{c}\text { Cavitación } \\
\text { incipiente }\end{array}$} & \multicolumn{2}{c|}{$\begin{array}{c}\text { Cavitación } \\
\text { severa }\end{array}$} \\
\cline { 2 - 7 } & Wav. & Time & Wav. & Time & Wav. & Time \\
\hline Caso 1 & 0,505 & 0,358 & 0,737 & 0,53 & 1,30 & 0,925 \\
\hline Caso 2 & 0,436 & 0,308 & 0,955 & 0,679 & 1,662 & 1,179 \\
\hline Caso 3 & 0,480 & 0,340 & 0,656 & 0,464 & 1,071 & 0,765 \\
\hline
\end{tabular}

\section{CONCLUSIONES}

En este artículo se propone y valida experimentalmente el uso de las emisiones acústicas para producir dos indicadores de falla, uno en dominio tiempo y el otro aplicando la transformada discreta de Wavelet, para el monitoreo del fenómeno de cavitación en bombas centrífugas. Las pruebas experimentales mostraron que los indicadores propuestos basados en la señal de emisiones acústicas no solo permiten la detección de la cavitación incipiente sino que permiten determinar el grado de severidad de ésta. Adicionalmente, ambos indicadores propuestos presentan una relación satisfactoria en cuanto a su cambio de magnitud ante el cambio en el nivel de severidad. Por lo tanto, es posible candidatizar a cualquiera de los indicadores propuestos como variable para monitorear la cavitación en una bomba centrífuga.

Aunque por motivos de espacio no se incluyen todos los resultados, la experimentación permitió evaluar la gran influencia que tiene la ubicación del sensor sobre la carcasa. Por lo tanto, la implementación de este esquema de monitoreo exige un estudio previo de la mejor ubicación del sensor en la carcasa de la bomba.

\section{REFERENCIAS}

[1] D. Mba, R.H. Bannister and G.E. Findlay. "Condition monitoring of low-speed rotating machinery using stress waves". Journal of Process Mechanical Engineering. Vol. 213, pp. 153-185. August, 1999. DOI: 10.1243/0954408991529906

[2] M.A.A. Elmaleeh, N.M. Saad, N. Ahmed and M. Awan. "On-line fault detection $\&$ diagnosis of rotating machines using acoustic emission monitoring techniques". International Conference on Intelligent and Advanced Systems, 2007. ICIAS 2007, pp. 897-900. November, 2007.

[3] X. Escaler, E. Egusquiza, M. Farhat, F. Avellan and M. Coussirat. "Detection of cavitation in hydraulic turbines". Mechanical systems and signal processing. Vol. 20, pp. 983-1007. August, 2006. DOI: 10.1016/j. ymssp.2004.08.006.

[4] L. Alfayez, D. Mba and G. Dyson. "The application of acoustic emission for detecting incipient cavitations and the best efficiency point of a $60 \mathrm{~kW}$ centrifugal pump: case study". NDT\&E international. December, 2004. DOI: 10.1016/j.ndteint.2004.10.002

[5] J.P. Dear and J.E. Field. "A Study of the collapse of arrays of cavities". Journal of Fluid Mechanics. Vol. 190, pp. 409-425. July, 1988. DOI: $10.1017 /$ S0022112088001387

[6] R.L. Mott. "Mecánica de Fluidos Aplicada". Prentice Hall. 5a Ed. New York, USA. 2006. ISBN: 9702608058.

[7] S. Cornelius and G. Paresh. "Practical Machinery Vibration Analysis and Predictive Maintenance". Elsevier, pp. 130131. Burlington, USA. 2004. ISBN: 978-0-7506-6275-8

[8] M. Lorenc, M. Szmechta, D. Zmarzly and T. Boczar. "Acoustic Emission Spectral Analysis of Ultrasound Induced Cavitation in Insulating Oil”. Proceedings of 2008 International Symposium on Electrical Insulating Materials, pp. 452-455. Japan. September, 2008. DOI: 10.1109/ISEIM.2008.4664583. 
[9] T. Boczar, M. Szmechta and D. Zmarzly. "Acoustic Emission Measurements of Acoustically Induced Cavitation Bubbles in Insulating Oils". International Conference on Dielectric Liquids, ICDL 2008 IEEE, pp. 1-4. July, 2008.
[10] C.K. Chui. "Wavelets: a Mathematical Tool for Signal Analysis". SIAM, p. 210. Philadelphia, USA. 1997. ISBN: 0898713846.

[11] I. Daubechies. "Ten Lectures on Wavelets". SIAM, p. 357. Philadelphia, USA. 1992. ISBN: 0898712742, 9780898712742. 